\title{
Application of Laboratory Atomic Physics to Some Significant Stellar Chemical Composition Questions
}

\author{
Christopher Sneden ${ }^{1}{ }^{(\mathbb{D}}$, James E. Lawler ${ }^{2}$ and Michael P. Wood ${ }^{3, *}$ \\ 1 Department of Astronomy and McDonald Observatory, The University of Texas, Austin, TX 78712, USA; \\ chris@verdi.as.utexas.edu \\ 2 Department of Physics, University of Wisconsin-Madison, 1150 University Ave, Madison, WI 53706, USA; \\ jelawler@wisc.edu \\ 3 Department of Physics, University of St. Thomas, 2115 Summit Ave, St. Paul, MN 55105, USA \\ * Correspondence: mpwood@stthomas.edu or chris@verdi.as.utexas.edu; Tel.: +1-512-471-1349
}

Received: 27 July 2018; Accepted: 23 August 2018; Published: 27 August 2018

\begin{abstract}
This brief review highlights some current issues in Galactic stellar nucleosynthesis, and some recent laboratory studies by the Wisconsin atomic physics group that have direct application to stellar spectroscopy to advance our understanding of the chemical evolution of our Galaxy. The relevant publication history of the lab studies are summarized, and investigations into the abundances of neutron-capture and iron-peak elements in low metallicity stars are described. Finally, new initiatives in near-infrared spectroscopy are briefly explored.
\end{abstract}

Keywords: atomic physics; transition probabilities; hyperfine and isotopic substructure; stellar spectroscopy; chemical compositions

\section{Introduction}

Fifteen years ago, the United States National Academy of Sciences suggested a set of "Eleven Science Questions for the New Century" [1]. One consideration deemed worthy of inclusion in that list was, "How Were the Elements from Iron to Uranium Made?" This question sums up the overall goals of many efforts in fields labeled variously as Galactic chemical evolution, stellar nucleosynthesis, and cosmochronology. Most information for these fields must come from stellar surface chemical compositions, with additional contributions from solar system meteoritics, planetary nebulae, $\mathrm{H}_{2}$ regions, and supernova remnants.

The basic tool for stellar elemental abundance studies is spectroscopy, generally obtained with wavelength resolving power comparable to the widths of stellar absorption lines. Most of the stars investigated for clues to nucleosynthesis are relatively cool, with effective temperatures $T_{\text {eff }}<10,000 \mathrm{~K}$. Their spectra are comprised almost entirely of neutral and singly-ionized species of elements with atomic numbers $Z \geq 6$ and diatomic molecules mostly involving the HCNO elements. Their surface chemical compositions reflect the combination of elements synthesized and ejected into the interstellar medium by past stellar generations, and elements produced in the interiors of the stars themselves.

Determination of accurate elemental abundances from atomic lines in stellar atmospheres involves many areas of physics and astronomy, including laboratory and theoretical atomic physics, statistical and thermal physics, construction of realistic model stellar photospheres, solutions to the radiation transport problem due to a variety of absorption and scattering processes, details of stellar line formation, etc. Limits on the abundance accuracies can arise from multiple sources, and any weak link in the analysis chain can potentially destroy the usefulness of an element in helping to understand nucleosynthesis. The quest for more accurate stellar abundances must start with the parameters of individual atomic transitions. One must know their quantum properties very well, including their 
transition probabilities and isotopic and hyperfine substructures, to have any hope of deriving robust abundances from them.

The Vienna Atomic Line Database (VALD; [2] and references therein; http:/ /vald.astro.uu.se/) is a major compilation of atomic transitions that are very useful to stellar spectroscopists. Many VALD data entries are from laboratory sources, but perhaps the majority of transitions come from the vast atomic and molecular line compendium of Kurucz ([3,4]; http:/ / kurucz.harvard.edu/), which contains a mix of laboratory, theoretical, and semi-empirical transition probabilities.

These databases also have estimates of line damping parameters; these are important in deriving accurate abundances for absorption lines with large optical depths. Some progress has been made on practical application of collisional broadening theory (e.g., [5-7]), and further effort in this area should pay large dividends, especially in the less-studied infrared spectral domain.

Several atomic physics groups are making major contributions to improving transition data through many theoretical and laboratory approaches. These groups and some representative publications include, but are not limited to, those at: The University of Mons [8,9]; Imperial College London [10,11]; Lund University [12,13]; and Uppsala University [7,14]. Our collaboration from The University of Wisconsin-Madison and The University of Texas at Austin has concentrated on improving these basic line parameters through laboratory experiments, and applying these lab data to classes of stars most relevant to Galactic chemical evolution descriptions. This paper aims to highlight the University of Wisconsin laboratory studies of atomic species most relevant to cool-star spectroscopy.

\section{Laboratory Transition Data}

Two important astrophysical element groups have been featured: the Fe-peak elements $(21 \leq Z \leq 30)$, and the neutron-capture ( $n$-capture) elements $(Z>30)$. In Figure 1 , we show in periodic table format the elements with published Wisconsin lab $g f$ values, color-coded by the species that have been studied. Except for Xe I [15] and Ni II [16], all of the results include transitions that are readily accessible in the optical (3000-9500 $⿱$ ) ) and ultraviolet (UV: 2200-3000 $)$ ) spectral domains of cool stars ${ }^{1}$. There are many individual studies, and here we comment on a few aspects of these lab investigations.

Of primary importance is the ability of stellar spectroscopists to have access to single-source internally consistent transition data for as many lines as possible for a given species. A few decades ago, stellar abundance studies had to rely on a patchwork set of relatively inaccurate lab and theoretical line parameters, or to resort to differential analyses of stellar and solar spectra. Neither of these approaches could produce abundances that are accurate enough to meaningfully compare to stellar nucleosynthesis predictions. Happily, the huge increase in quality and quantity of transition data means that, for most spectroscopically-accessible species, the basic line data are now very small factors in abundance error budgets.

Among the Fe-group elements, accurate transition probabilities have been published recently for Fe I $[10,17,18]$ through the combined efforts of the groups at Imperial College London, Uppsala, and Wisconsin. Almost all of the transitions reported in these papers arise from states with lower excitation energies $\chi>2.2 \mathrm{eV}$. For Fe I lines with smaller excitation energies, the older lab study of $\mathrm{O}^{\prime}$ Brian et al. [19] still provides trustworthy transition probabilities. Combining lab data from these studies and applying them to the solar photosphere and metal-poor stars appears to yield reliable Fe abundances with relatively small line-to-line scatter [20]. Unfortunately, no single comprehensive lab study of Fe II has been published recently; see the summary of sources up to 2016 in [20].

The neutral species for Ti through Ni have been extensively studied [21-26], and the ionized species for these elements except Ni (see above footnote) [24,27-30]. Application to the solar photospheric spectrum and to the spectrum of the well-studied metal-poor field star HD 84937 results in accurate,

1 Only the Xe I $1470 \AA$ A resonance line was investigated by Anderson et al. [15]; seven Ni II lines in the 1450-1750 A were included by Fedchak and Lawler [16]. 
internally consistent abundances for each species. More importantly, the abundances for both species (when available) are in agreement (see Figure 7 of [20]), strongly suggesting that the derived Fe-group abundances are true chemical composition markers, relatively unaffected by line formation and stellar atmospheric modeling uncertainties.

An earlier series of lab studies concentrated on near-complete coverage of the ionized species of "rare earth" lanthanides. Only the ionized species of lanthanides are usually detectable in stellar spectra. Despite their relatively small abundances in most stars in comparison to the Fe-peak group, there are many ionized-species lanthanide lines detectable in the blue and near- $U V$ spectrum of cool stars. In Figure 1, it is clear that most Wisconsin lab studies of $n$-capture elements outside the lanthanide domain have been on neutral-species transitions. In many cases, this mirrors their stellar spectroscopic accessibility. Neutral species lines of these elements mostly are few and far between, and rarely do ionized species lines become detectable.

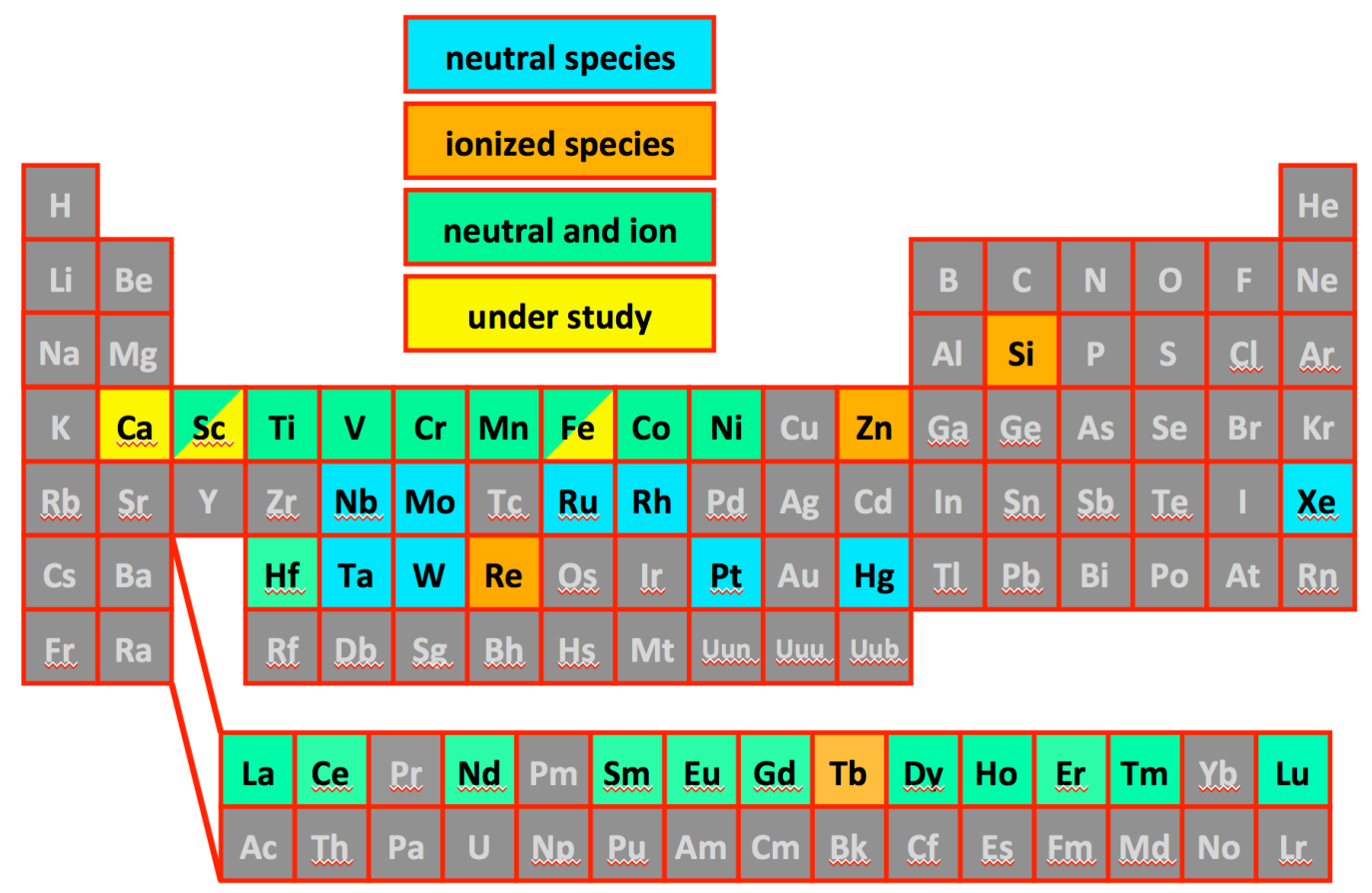

Figure 1. A periodic table color-coded by published neutral and ionized species laboratory transition probabilities from the Wisconsin group. The elements shown in gray boxes have not been studied; the blue, orange, green and yellow colors are defined in the figure.

\section{Some Applications of Laboratory Data to Low Metallicity Stars}

The onset of Galactic nucleosynthesis produced stars of very low overall metallicity ${ }^{2}$. Many of these objects exhibit large departures from the solar-system abundance distribution. Among the $n$-capture elements, there are outstanding examples of stars that have been born with or acquired from a companion extreme enhancements of elements (isotopes) that were generated in fusion environments that were conducive to slow neutron captures (the s-process) or rapid-blast ones (the $r$-process).

There has been renewed interest in the $r$-process production site following the recent discovery of gravitational wave event GW170817, so far unique in providing electromagnetic signals as well in multiple wavelength domains. The totality of "multi-messenger" information from GW170817 strongly

2 We adopt the standard spectroscopic notation [31] that, for elements $A$ and $B,[A / B] \equiv \log _{10}\left(N_{A} / N_{B}\right)_{\star}-\log _{10}\left(N_{A} / N_{B}\right)_{\odot}$. We use the definition $\log \epsilon(\mathrm{A}) \equiv \log _{10}\left(\mathrm{~N}_{\mathrm{A}} / \mathrm{N}_{\mathrm{H}}\right)+12.0$, and equate metallicity with the stellar $[\mathrm{Fe} / \mathrm{H}]$ value. 
suggests that this event arose from the merger of two neutron stars [32]. Additionally, photometric and spectroscopic observations [33,34] suggest the presence of $r$-process-rich ejecta from this event, in particular of unstable lanthanide isotopes. It is important to determine accurate abundances for as many $n$-capture elements in $r$-process-rich stars as possible to quantitatively constrain what the output of a binary neutron star merger must match.

The first extremely $r$-process-rich star, CS 22892-052, was recognized on a noisy high-resolution spectrum and analyzed with weak laboratory data [35]. A total of 54 lines contributed to abundances of $12 n$-capture species. Contrast that with the study of the recently-discovered $r$-process-rich star J09544277+5246414 (J0954+5246) [36], which reported abundances based on 134 lines of 28 species. In Figure 2, we reproduce their Figure 1, a comparison of abundances of that star and scaled solar-system $r$-process-only abundances. The bottom panel of this figure also includes in the $n$-capture abundances for CS 31082-001 [37], a very metal-poor $r$-process-rich star. This Galactic halo member is notable especially for its Th and $U$ abundances, which are larger than the extrapolated scaled solar-system values. CS 31082-001 became the first of such "actinide boost" stars, which now includes J0954+5246.

For the present review, a more important point is the match between the abundance distribution in the lanthanide domain to the solar-system $r$-process-only abundances. It is near-perfect for the two stars in Figure 2 and in many other $r$-process-rich stars (e.g., [38,39]). Spectroscopically, this means that atomic data parameters for a large number of detectable $n$-capture elements are now good enough that they play only a very small part of the overall elemental abundance uncertainties. Astrophysically, this means that the creation of $r$-process elements has been essentially the same over Galactic history, and possibly limited to one type of production site.

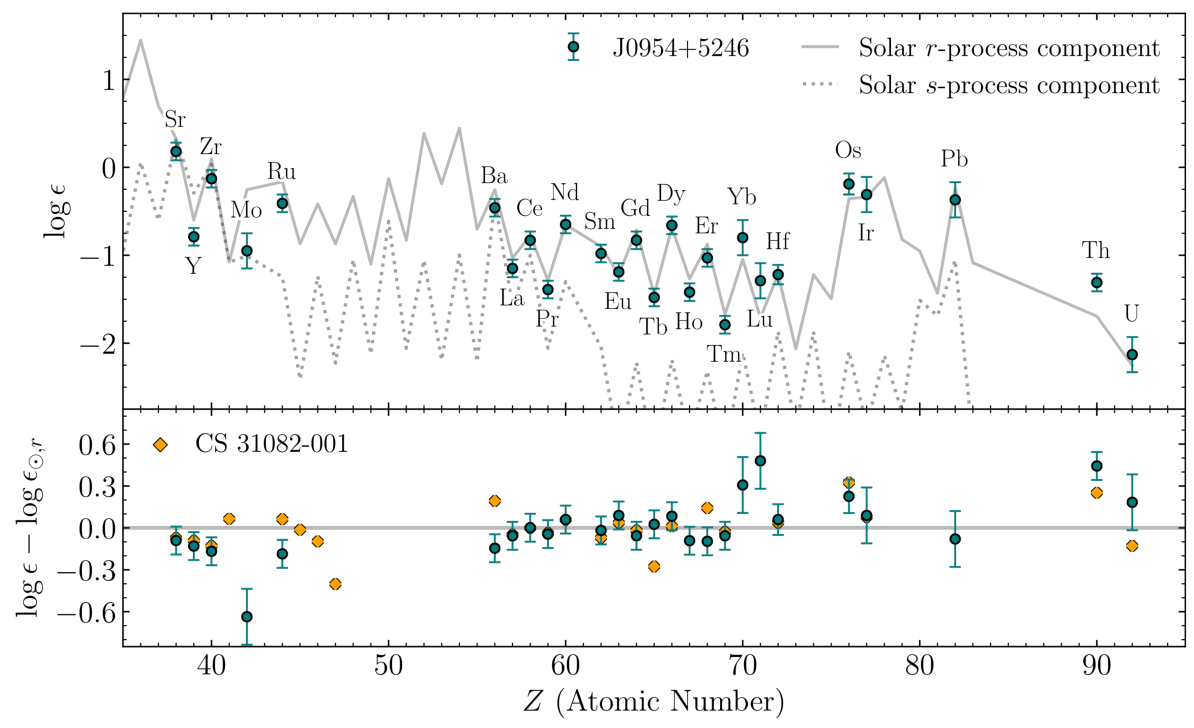

Figure 2. Abundances of $n$-capture elements in the very metal-poor $r$-process-rich stars J0954+5246, CS 31082-001, and $r$-process and s-process material in the solar system ([36]; their Figure 1). Abundances for CS 31082-001 are taken from [37]: (Top) the abundances; and (Bottom) the differences between the star and solar $r$-process-only abundances are shown.

For low metallicity stars, the Fe-group element abundances have not had the same level of atomic physics attention as the $n$-capture elements. It has been known for decades that relative abundances among this element group do not conform exactly to the solar-system distribution [40-42]; [Mn/Fe] $<0$ and $[\mathrm{Cu} / \mathrm{Fe}]<0$. Additionally, McWilliam et al. [43], Cayrel et al. [44] suggested that, below metallicity levels of $[\mathrm{Fe} / \mathrm{H}] \sim-2.5$, there are decreases in $[\mathrm{Cr} / \mathrm{Fe}]$ and increases in $[\mathrm{Co} / \mathrm{Fe}]$, yielding $[\mathrm{Co} / \mathrm{Cr}] \sim+1$. However, these results have generally depended on very few transitions of the neutral (minority) species of $\mathrm{Mn}, \mathrm{Cr}, \mathrm{Co}$, and $\mathrm{Cu}$. Recently, with the new Wisconsin lab transition data discussed in 
Section 2, a large study of a warm metal-poor $([\mathrm{Fe} / \mathrm{H}] \sim-2.3)$ unevolved "main sequence" turnoff star [20] included these features:

- $\quad$ continuous spectral coverage from the $U V$ through the optical, 2300-8000 $\AA$;

- $\quad \sim 550 \mathrm{Fe}$ I and Fe II lines, 600 lines of other Fe-group neutral and ionized species ([20] Figure 1);

- precise abundances from $17 \mathrm{Fe}$-peak species (mean line-to-line scatters $\langle\sigma \log \epsilon\rangle=0.07$ ), with good agreement (mean $\log \epsilon$ differences of 0.03 dex) between neutrals and ions of seven elements ([20] Figure 7);

- little sign of over-ionization of neutral species, which would be a signature of departures from local thermodynamic equilibrium (LTE) in the Fe-group neutral species, which could be a fortuitous result that can be realized as well by a combination of more realistic three-dimensional model atmospheres and detailed non-LTE computations (e.g., [45]);

- $\quad$ no evidence for depletion of $\mathrm{Cr}$ and enhancement of $\mathrm{Co}$ at this metallicity; and

- a curious overabundance of the three lightest Fe-peak elements Sc, Ti, and V.

This work is being extended to lower metallicities $[46,47]$ to investigate Fe-peak abundance ratios for any solid evidence of other departures from the solar-system distribution.

Lab studies of Fe-peak elements are continuing. The HD 84937 study detailed above did not include recent transition studies of Sc II, Cr II, Co II, Cu I or Zn I. New data for Cr II [28] yields consistent abundances from neutral and ionized species in the solar photosphere. The same agreement is seen in HD 84937 as long as the anomalously small abundances (0.2-0.3 dex lower than the rest of the Cr transitions) from the Cr I resonance lines at 4254,4274 , and $4289 \AA$ are ignored. This appears to be a clear signal of departures from LTE in the neutral species. We suspect that this may explain the earlier reports of $\mathrm{Cr}$ deficiencies in very metal-poor stars, since the $\mathrm{Cr}$ I resonance lines have played a major role in most Fe-peak abundance surveys.

Recently, Lawler et al. [29] reported new lab data for Co II transitions, almost all of which arise in the $U V$ spectral region. In Figure 3, we summarize the HD 84937 abundances from that study. Translating these $\log \epsilon$ values into relative abundances with respect to the Sun yields $[\mathrm{Co} / \mathrm{H}]=-2.24$ or $[\mathrm{Co} / \mathrm{Fe}]=+0.08$, essentially the solar ratio within the observational uncertainties. There is no obvious enhancement in the relative Co abundance at this metallicity. Interestingly, the abundance from Co I lines is 0.08 larger; one looks for over-ionization of an element as evidence for non-LTE effects, but in HD 84937 we derive an under-ionization. This anomaly is being further tested with our more metal-poor stellar sample [46,47]; preliminary evidence supports the notion that Co is not overabundant in very metal-poor stars, i.e., $[\mathrm{Co} / \mathrm{Fe}] \sim 0$ for stars with $[\mathrm{Fe} / \mathrm{H}] \leq-3$.

The vacuum- $U V$ spectral region, $\lambda<3000 \AA$, presents the ideal stellar laboratory to make progress in understanding Fe-peak abundances in low metallicity stars. Hundreds of potentially useful neutral and ionized species transitions can be easily identified in the vacuum $U V$. Unfortunately, the required high-resolution spectra may only be acquired with the Hubble Space Telescope STIS instrument. At present, there are less than about 20 metal-poor $([\mathrm{Fe} / \mathrm{H}]<-2)$ stars with good HST / STIS UV spectra. Given the ongoing demand for HST time, one cannot reasonably expect a large expansion of this meager database. A more feasible goal would be to use ground-based large telescopes with good near-UV high resolution spectrographs (e.g., VLT /UVES and Keck/HIRES) to access more Fe-peak transitions in the 3000-4000 ̊̊ spectral range. 

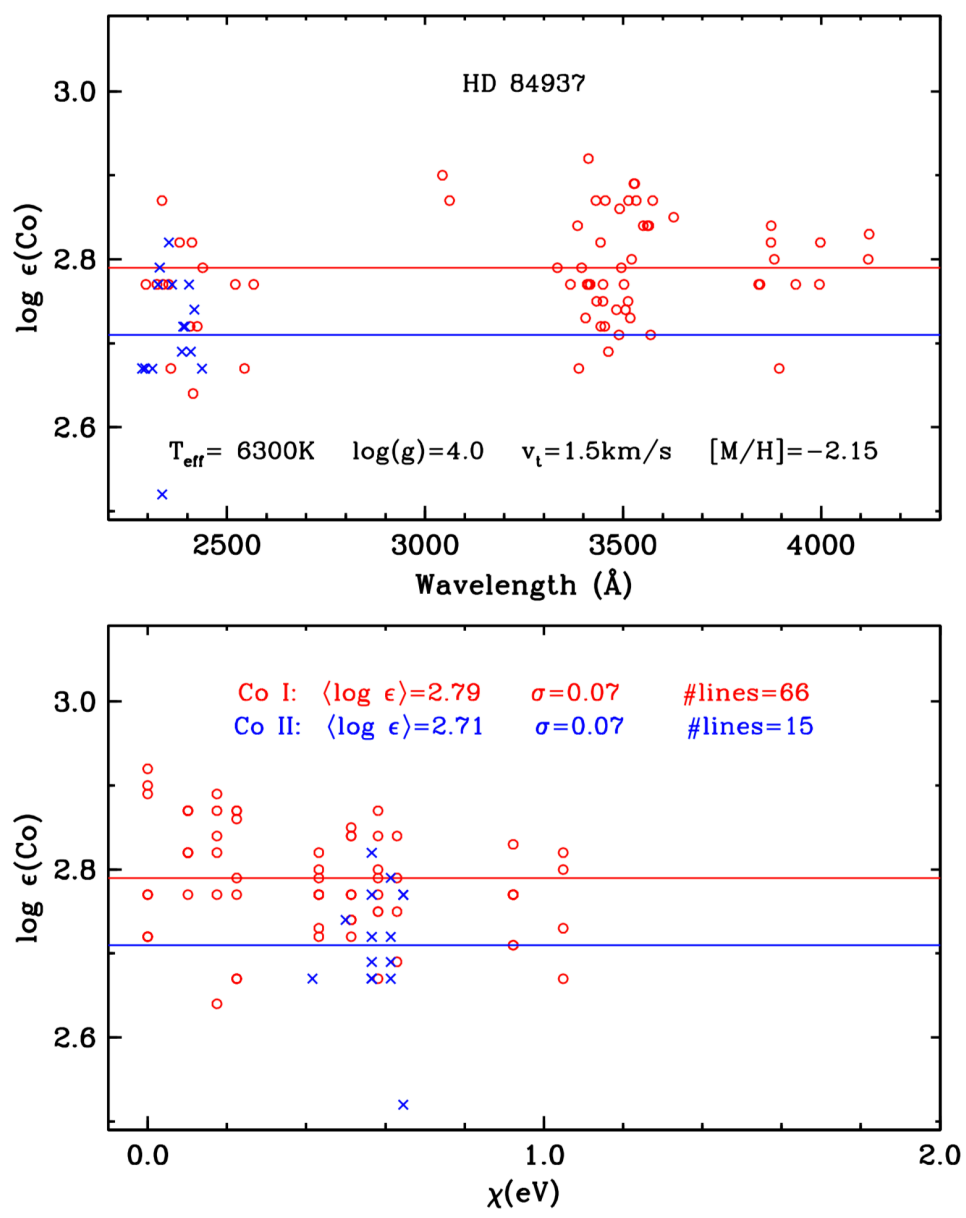

Figure 3. Abundances of Co I (red circles) and Co II (blue x's) lines in HD 84937 as functions of: wavelength (Top); and and excitation energy (Bottom) (taken from [29]).

\section{Extending the Wavelength Domain to the Near-Infrared}

The near-infrared spectral domain, 1.0-2.4 $\mu \mathrm{m}$, is enjoying new high-resolution spectroscopic access, with such telescope/instrument combinations as VLT/CRIRES, SDSS/APOGEE, and McDonald - DCT - Gemini / IGRINS now able to provide quality spectra of many faint $(K \leq 14)$ targets in star clusters, the general Milky Way field and the Galactic bulge. Laboratory atomic physics so far has not been able to keep pace in the near- $I R$, mostly due to intrinsic difficulties in deriving accurate atomic transition probabilities. Upper levels that only radiate in the infrared $(I R)$ are not currently accessible to TRLIF (Time-Resolved Laser-Induced Fluorescence) radiative lifetime measurements. The lack of an IR equivalent to an optical-UV photomultiplier (PMT) is a problem. The combination of $P M T$ gain, electronic bandwidth, and detector area is not yet available in the IR. Progress is needed, and here we describe some aspects of the efforts of the Wisconsin group in this spectral domain.

Lawler et al. [21] reported accurate transition probabilities for nearly $950 \mathrm{Ti}$ I lines, and derived new $\mathrm{Ti}$ abundances for the solar photosphere and very metal-poor star HD 84937. However, the absorption strengths of Ti I lines, similar to those of most species, decrease substantially with increasing wavelength, and there are relatively few measurable ones in warm stars such as the Sun and especially in metal-poor stars. Such stars cannot be used to test the parameters of many atomic lines redward of $\sim 8000 \AA$ A. For this task, we have recently turned to the bright, mildly metal-poor $([\mathrm{Fe} / \mathrm{H}] \simeq-0.5)$ red giant star Arcturus. This star has both excellent high-resolution spectroscopic atlases and careful model atmosphere studies. The reader is referred to [48] for citations to these studies and a discussion of V I transitions in the red spectral region of Arcturus. In Figure 4, we present abundances for $168 \mathrm{Ti}$ I lines in Arcturus from $5200 \AA$ to $2.4 \mu \mathrm{m}$. The mean abundance, $\log \epsilon(\mathrm{Ti}) \simeq 4.6$, suggests that $[\mathrm{Ti} / \mathrm{H}] \simeq-0.4$ 
or $[\mathrm{Ti} / \mathrm{Fe}] \simeq+0.1$. The transition probabilities for these analyses all come from [21], and in general they yield a consistent Ti abundance. There is a very small upward drift in derived abundances with increasing wavelength, but it may arise from a variety of possible causes. The important conclusion is that for $\mathrm{Ti}$ I the optical and near- $I R$ data essentially agree, lending confidence to the lab transition probabilities for the long wavelength lines.

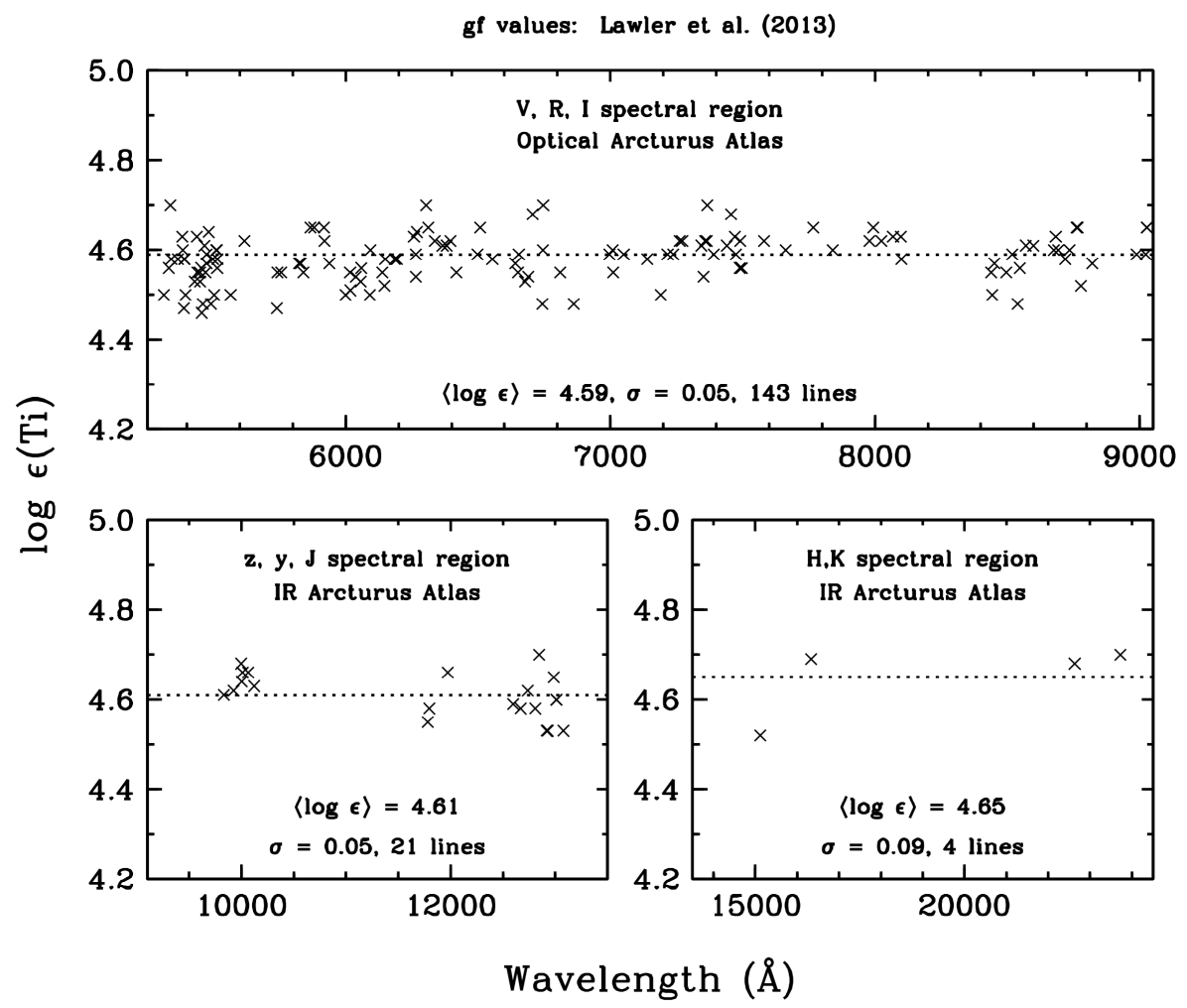

Figure 4. Abundances of Ti in the well-studied red giant Arcturus from Ti I lines in the optical and near- $I R$ spectral domains: (Top) results from the optical region defined by astronomical photometric bands $V, R$, and $I$; and (Bottom) near- $I R$ spectral regions $z, y$, and $J$ (left); and $H$ and $K$ (right). Obvious wavelength gaps with no Ti I line abundances are mostly due to telluric molecular absorption blocking starlight from reaching the ground.

Stellar spectroscopists usually focus on using the best available lab $g f$-values for abundance work. However, as the spectroscopic datasets attain ever-increasing resolution and signal-to-noise quality, some secondary atomic data considerations become important for the most accurate abundances. Odd- $Z$ elements and odd- $A$ isotopes often yield transitions with significant hyperfine structure. Examples of this have been well documented in recent Fe-peak studies [22,28-30]. Less attention has been paid to isotopic displacement of spectral lines of some elements. The isotopic wavelength offsets are undetectably small in the $U V$ and most of the optical spectral region, but they grow with increasing wavelength.

$\mathrm{Ni}$ has five naturally-occurring isotopes, three of which have non-negligible percentages in solar-system material: ${ }^{58} \mathrm{Ni}, 68.1 \% ;{ }^{60} \mathrm{Ni}, 26.2 \%$; and ${ }^{62} \mathrm{Ni}, 3.6 \%$. For $\mathrm{Ni}$ I, the presence of significant isotopic splitting for several lines redward of $7000 \AA$ has been demonstrated by Wood et al. [26] (see their Figure 10). Hyperfine and isotopic subcomponents of spectral lines will always act to spread the absorption in wavelength, desaturating the overall feature. Neglect of transition substructure can lead to over-estimation of abundances, and for strong lines this can be a significant effect.

Examination of the solar photospheric spectrum [49] has called attention of the isotopic substructure of Ti I transitions, which have been previously reported in laboratory studies (e.g., [50]). In solar-system material, Ti splits into these isotopes: ${ }^{46} \mathrm{Ti}, 8.25 \% ;{ }^{47} \mathrm{Ti}, 7.44 \% ;{ }^{48} \mathrm{Ti}, 73.72 \%$; ${ }^{49} \mathrm{Ti}, 5.41 \%$; and ${ }^{50} \mathrm{Ti}, 5.18 \%$. 
The isotopic broadening of optical Ti I lines is negligible, but detectable in the near-IR. As an example, in Figure 5, we show one near- $I R$ transition in the lab and in the solar photosphere. Isotopic wavelength shifts are not resolved into individual components, but the extra line broadening is evident. As with $\mathrm{Ni}$, this effect can become important in deriving accurate elemental abundances when Ti I lines become strong, as they surely will in cool red giant stellar spectra. Note finally that detection of isotopes in stellar line broadening does not generally lead to much leverage in determining the isotopic fractions for most elements. For Ti, it is more profitable to do isotopic studies with molecular TiO, as in [51].
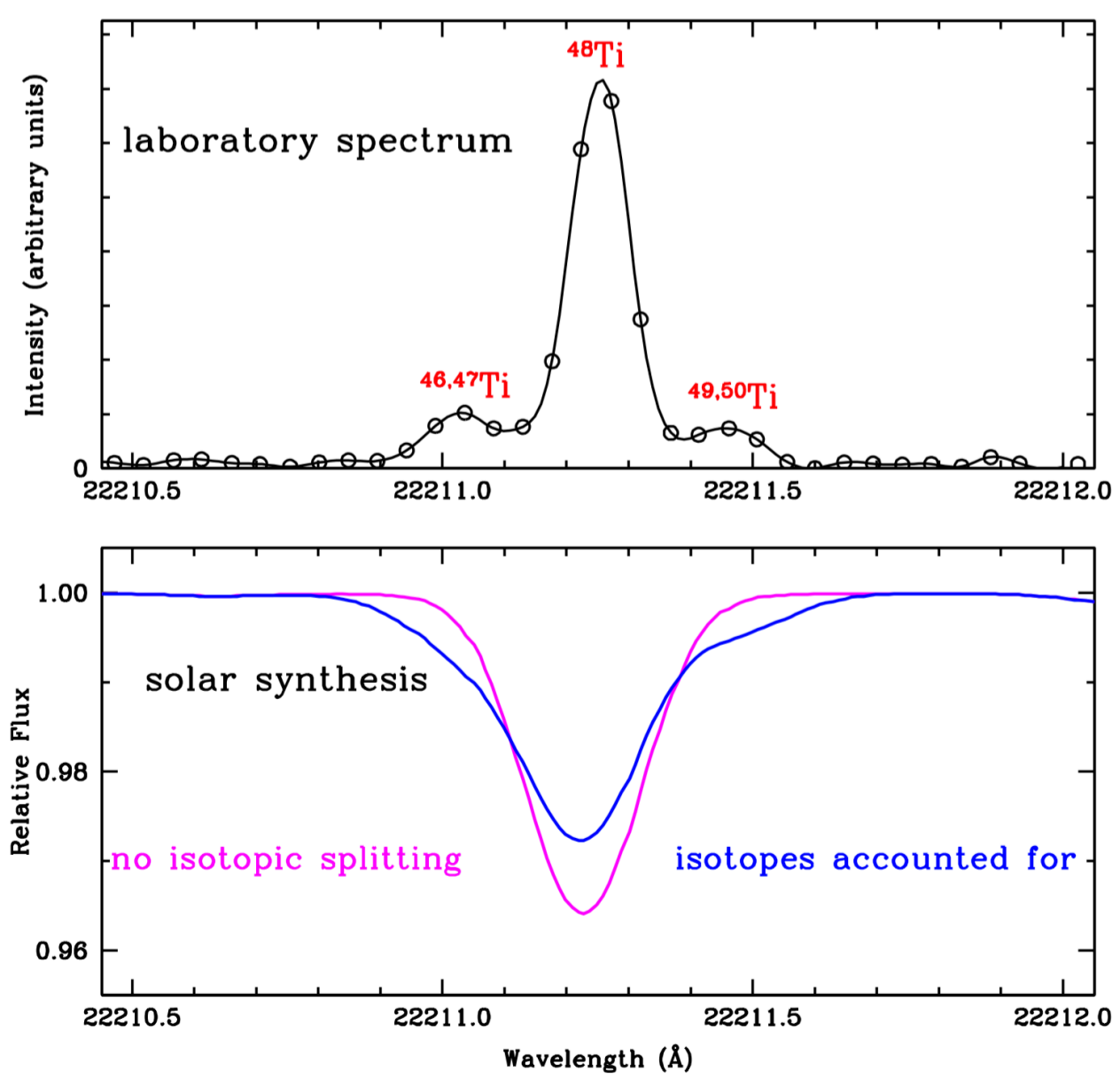

Figure 5. An example of isotopic broadening in Ti I transitions: (Top) a laboratory emission spectrum of one of the near- $I R$ lines (see [49] for details); and (Bottom) synthetic solar profiles for this transition with (blue) and without (magenta) proper accounting for the minor isotopes of Ti.

Author Contributions: Conceptualization, J.E.L. and C.S.; Methodology, J.E.L., C.S. and M.P.W.; Software, J.E.L., C.S. and M.P.W.; Validation, J.E.L., C.S. and M.P.W.; Formal Analysis, J.E.L., C.S. and M.P.W.; Resources, J.E.L. and C.S.; Data Curation, J.E.L., C.S. and M.P.W.; Writing-Original Draft Preparation, C.S.; Writing-Review and Editing, J.E.L., C.S. and M.P.W.; Visualization, C.S.; Project Administration, J.E.L. and C.S.; and Funding Acquisition, J.E.L. and C.S.

Funding: We are happy to acknowledge support by NASA grant NNX16AE96G (J.E.L.) and by NSF grant AST1616040 (C.S.).

Acknowledgments: We thank all of our colleagues in atomic physics and stellar spectroscopy who have contributed over decades to the work summarized in this paper.

Conflicts of Interest: The authors declare no conflict of interest. 


\section{References}

1. Committee on the Physics of the Universe; Board on Physics and Astronomy; Division on Engineering and Physical Sciences; National Research Council of the National Academies. Connecting Quarks with the Cosmos: Eleven Science Questions for the New Century; National Academies Press: Washington, DC, USA, 2003.

2. Ryabchikova, T.; Piskunov, N.; Kurucz, R.L.; Stempels, H.C.; Heiter, U.; Pakhomov, Y.; Barklem, P.S. A major upgrade of the VALD database. Phys. Scr. 2015, 90, 054005. [CrossRef]

3. Kurucz, R.L. Including all the lines. Can. J. Phys. 2011, 89, 417-428. [CrossRef]

4. Kurucz, R.L. Problems with Atomic and Molecular Data: Including All the Lines. In Determination of Atmospheric Parameters of B-, A-, F- and G-Type Stars; Niemczura, E., Smalley, B., Pych, W., Eds.; Springer: Cham, Switzerland, 2014; pp. 63-73, ISBN 978-3-319-06955-5.

5. Anstee, S.D.; O'Mara, B.J. Width cross-sections for collisional broadening of s-p and p-s transitions by atomic hydrogen. Mon. Not. R. Astron. Soc. 1995, 276, 859-866. [CrossRef]

6. Barklem, P.S.; Piskunov, N.; O'Mara, B.J. A list of data for the broadening of metallic lines by neutral hydrogen collisions. Astron. Astrophys. Suppl. 2000, 142, 467-473. [CrossRef]

7. Barklem, P.S. Accurate abundance analysis of late-type stars: advances in atomic physics. Astron. Astrophys. Rev. 2016, 24, 9:1-9:54. [CrossRef]

8. Pehlivan Rhodin, A.; Belmonte, M.T.; Engström, L.; Lundberg, H.; Nilsson, H.; Hartman, H.; Pickering, J.C.; Clear, C.; Quinet, P.; Fivet, V.; et al. Lifetime measurements and oscillator strengths in singly ionized scandium and the solar abundance of scandium. Mon. Not. R. Astron. Soc. 2017, 472, 3337-3353. [CrossRef]

9. Quinet, P.; Fivet, V.; Palmeri, P.; Engström, L.; Hartman, H.; Lundberg, H.; Nilsson, H. Experimental radiative lifetimes for highly excited states and calculated oscillator strengths for lines of astrophysical interest in singly ionized cobalt (Co II). Mon. Not. R. Astron. Soc. 2016, 462, 3912-3917. [CrossRef]

10. Belmonte, M.T.; Pickering, J.C.; Ruffoni, M.P.; Den Hartog, E.A.; Lawler, J.E.; Guzman, A.; Heiter, U. Fe I Oscillator Strengths for Transitions from High-lying Odd-parity Levels. Mon. Not. R. Astron. Soc. 2017, 848, 125. [CrossRef]

11. Belmonte, M.T.; Pickering, J.C.; Clear, C.; Liggings, F.; Thorne, A.P. Accurate atomic data for Galactic Surveys. In Proceedings of the IAU Symposium S330: Astrometry and Astrophysics in the Gaia Sky, Nice, France, 24-28 April 2017; Recio-Blanco, A., de Laverny, P., Brown, A.G.A., Prusti, T., Eds.; Cambridge University Press: Cambridge, UK, 2017; pp. 203-205.

12. Engström, L.; Lundberg, H.; Nilsson, H.; Hartman, H.; Bäckström, E. The FERRUM project: Experimental transition probabilities from highly excited even 5 s levels in Cr II. Astron. Astrophys. 2014, 570, A34:1-A34:5. [CrossRef]

13. Hartman, H.; Engström, L.; Lundberg, H.; Nilsson, H.; Quinet, P.; Fivet, V.; Palmeri, P.; Malcheva, G.; Blagoev, K. Radiative data for highly excited $3 \mathrm{~d}^{8} 4 \mathrm{~d}$ levels in Ni II from laboratory measurements and atomic calculations. Astron. Astrohys. 2017, 600, A108:1-A108:13. [CrossRef]

14. Rodionov, D.S.; Belyaev, A.K.; Guitou, M.; Spielfiedel, A.; Feautrier, N.; Barklem, P.S. Inelastic cross sections for low-energy Mg + H collisions. J. Phys. Conf. Ser. 2014, 572, 012010:1-012010:7. [CrossRef]

15. Anderson, H.M.; Bergeson, S.D.; Doughty, D.A.; Lawler, J.E. Xenon 147-nm resonance f value and trapped decay rates. Phys. Rev. A 1995, 51, 211-217. [CrossRef] [PubMed]

16. Fedchak, J.A.; Lawler, J.E. Absolute UV And Vacuum UV Oscillator Strengths For Ni II. Astrophys. J. 1999, 523, 734-738. [CrossRef]

17. Ruffoni, M.P.; Den Hartog, E.A.; Lawler, J.E.; Brewer, N.R.; Lind, K.; Nave, G.; Pickering, J.C. Fe I oscillator strengths for the Gaia-ESO survey. Mon. Not. R. Astron. Soc. 2014, 441, 3127-3136. [CrossRef]

18. Den Hartog, E.A.; Ruffoni, M.P.; Lawler, J.E.; Pickering, J.C.; Lind, K.; Brewer, N.R. Fe I Oscillator Strengths for Transitions from High-lying Even-parity Levels. Astrophys. J. Suppl. Ser. 2014, 215, 23:1-23:13. [CrossRef]

19. O’Brian, T.R.; Wickliffe, M.E.; Lawler, J.E.; Whaling, W.; Brault, J.W. Lifetimes, transition probabilities, and level energies in Fe i. J. Opt. Soc. Am. B 1991, 8, 1185-1201. [CrossRef]

20. Sneden, C.; Cowan, J.J.; Kobayashi, C.; Pignatari, M.; Lawler, J.E.; Den Hartog, E.A.; Wood, M.P. Iron-group Abundances in the Metal-poor Main-Sequence Turnoff Star HD 84937. Astrophys. J. 2016, 817, 53:1-53:16. [CrossRef] 
21. Lawler, J.E.; Guzman, A.; Wood, M.P.; Sneden, C.; Cowan, J.J. Improved Log(gf) Values for Lines of Ti I and Abundance Determinations in the Photospheres of the Sun and Metal-Poor Star HD 84937 (Accurate Transition Probabilities for Ti I). Astrophys. J. Suppl. Ser. 2013, 205, 11:1-11:15. [CrossRef]

22. Lawler, J.E.; Wood, M.P.; Den Hartog, E.A.; Feigenson, T.; Sneden, C.; Cowan, J.J. Improved V I Log(gf) Values and Abundance Determinations in the Photospheres of the Sun and Metal-poor Star HD 84937. Astrophys. J. Suppl. Ser. 2014, 215, 20:1-20:13. [CrossRef]

23. Sobeck, J.S.; Lawler, J.E.; Sneden, C. Improved Laboratory Transition Probabilities for Neutral Chromium and Redetermination of the Chromium Abundance for the Sun and Three Stars. Astrophys. J. 2007, 667, 1267-1282. [CrossRef]

24. Den Hartog, E.A.; Lawler, J.E.; Sobeck, J.S.; Sneden, C.; Cowan, J.J. Improved log(gf ) Values of Selected Lines in Mn I and Mn II for Abundance Determinations in FGK Dwarfs and Giants. Astrophys. J. Suppl. Ser. 2011, 194, 35:1-35:11. [CrossRef]

25. Lawler, J.E.; Sneden, C.; Cowan, J.J. Improved Co I log(gf) Values and Abundance Determinations in the Photospheres of the Sun and Metal-poor Star HD 84937. Astrophys. J. Suppl. Ser. 2015, 220, 13:1-13:11. [CrossRef]

26. Wood, M.P.; Lawler, J.E.; Sneden, C.; Cowan, J.J. Improved Ni I log(gf) Values and Abundance Determinations in the Photospheres of the Sun and Metal-poor Star HD 84937. Astrophys. J. Suppl. Ser. 2014, 211, 20:1-20:13. [CrossRef]

27. Wood, M.P.; Lawler, J.E.; Sneden, C.; Cowan, J.J. Improved Ti II Log(gf) Values and Abundance Determinations in the Photospheres of the Sun and Metal-Poor Star HD 84937. Astrophys. J. Suppl. Ser. 2013, 208, 27:1-27:10. [CrossRef]

28. Lawler, J.E.; Sneden, C.; Nave, G.; Den Hartog, E.A.; Emrahoğlu, N.; Cowan, J.J. Improved Cr II log(gf) Values and Abundance Determinations in the Photospheres of the Sun and Metal-poor Star HD 84937. Astrophys. J. Suppl. Ser. 2017, 228, 10:1-10:16. [CrossRef] [PubMed]

29. Lawler, J.E.; Feigenson, T.; Sneden, C.; Cowan, J.J.; Nave, G. Transition probabilities of Co II, weak lines to the ground and low metastable levels. arXiv 2018, arXiv:astro-ph.SR/1806.00581.

30. Wood, M.P.; Lawler, J.E.; Den Hartog, E.A.; Sneden, C.; Cowan, J.J. Improved V II Log(gf) Values, Hyperfine Structure Constants, and Abundance Determinations in the Photospheres of the Sun and Metal-poor Star HD 84937. Astrophys. J. Suppl. Ser. 2014, 214, 18:1-18:12. [CrossRef]

31. Wallerstein, G.; Helfer, H.L. Adundances in G Dwarf Stars. II. The High-Velocity Star 85 Pegasi. Astrophys. J. 1959, 129, 720-723. [CrossRef]

32. Abbott, B.P.; Abbott, R.; Abbott, T.D.; Acernese, F.; Ackley, K.; Adams, C.; Adams, T.; Addesso, P.; Adhikari, R.X.; Adya, V.B.; et al. GW170817: Observation of Gravitational Waves from a Binary Neutron Star Inspiral. Phys. Rev. Lett. 2017, 119, 161101:1-161101:18. [CrossRef] [PubMed]

33. Drout, M.R.; Piro, A.L.; Shappee, B.J.; Kilpatrick, C.D.; Simon, J.D.; Contreras, C.; Coulter, D.A.; Foley, R.J.; Siebert, M.R.; Morrell, N.; et al. Light curves of the neutron star merger GW170817/SSS17a: Implications for r-process nucleosynthesis. Science 2017, 358, 1570-1574. [CrossRef] [PubMed]

34. Shappee, B.J.; Simon, J.D.; Drout, M.R.; Piro, A.L.; Morrell, N.; Prieto, J.L.; Kasen, D.; Holoien, T.W.S.; Kollmeier, J.A.; Kelson, D.D.; et al. Early spectra of the gravitational wave source GW170817: Evolution of a neutron star merger. Science 2017, 358, 1574-1578. [CrossRef] [PubMed]

35. Sneden, C.; Preston, G.W.; McWilliam, A.; Searle, L. Ultrametal-poor halo stars: The remarkable spectrum of CS 22892-052. Astrophys. J. 1994, 431, L27-L30. [CrossRef]

36. Holmbeck, E.M.; Beers, T.C.; Roederer, I.U.; Placco, V.M.; Hansen, T.T.; Sakari, C.M.; Sneden, C.; Liu, C.; Lee, Y.S.; Cowan, J.J.; et al. The R-Process Alliance: 2MASS J09544277+5246414, the Most Actinide-enhanced R-II Star Known. Astrophys. J. 2018, 859, L24:1-L24:6. [CrossRef]

37. Hill, V.; Plez, B.; Cayrel, R.; Beers, T.C.; Nordström, B.; Andersen, J.; Spite, M.; Spite, F.; Barbuy, B.; Bonifacio, P.; et al. First stars. I. The extreme r-element rich, iron-poor halo giant CS 31082-001. Implications for the r-process site(s) and radioactive cosmochronology. Astron. Astrophys. 2002, 387, 560-579. [CrossRef]

38. Sneden, C.; Cowan, J.J.; Gallino, R. Neutron-Capture Elements in the Early Galaxy. Annu. Rev. Astron. Astrophys. 2008, 46, 241-288. [CrossRef]

39. Siqueira Mello, C.; Spite, M.; Barbuy, B.; Spite, F.; Caffau, E.; Hill, V.; Wanajo, S.; Primas, F.; Plez, B.; Cayrel, R.; et al. First stars. XVI. HST/STIS abundances of heavy elements in the ura nium-rich metal-poor star CS 31082-001. Astron. Astrophys. 2013, 550, A122:1-A122:17. [CrossRef] 
40. Lambert, D.L. The chemical composition of main sequence stars. In Proceedings of the 1998 AIP Conference on Cosmic Abundances of Matter, Minneapolis, MN, USA, 15-17 August 1988; Waddington, C.J., Ed.; Volume 183, pp. 168-199.

41. Wheeler, J.C.; Sneden, C.; Truran, J.W., Jr. Abundance ratios as a function of metallicity. Annu. Rev. Astron. Astrophys. 1989, 27, 279-349. [CrossRef]

42. Sneden, C.; Gratton, R.G.; Crocker, D.A. Trends in copper and zinc abundances for disk and halo stars. Astron. Astrophys. 1991, 246, 354-367.

43. McWilliam, A.; Preston, G.W.; Sneden, C.; Searle, L. Spectroscopic Analysis of 33 of the Most Metal Poor Stars. II. Astron. J. 1995, 109, 2757-2799. [CrossRef]

44. Cayrel, R.; Depagne, E.; Spite, M.; Hill, V.; Spite, F.; François, P.; Plez, B.; Beers, T.; Primas, F.; Andersen, J.; et al. First stars V-Abundance patterns from $\mathrm{C}$ to $\mathrm{Zn}$ and supernova yields in the early Galaxy. Astron. Astrophys. 2004, 416, 1117-1138. [CrossRef]

45. Amarsi, A.M.; Lind, K.; Asplund, M.; Barklem, P.S.; Collet, R. Non-LTE line formation of Fe in late-type stars-III. 3D non-LTE analysis of metal-poor stars. Mon. Not. R. Astron. Soc. 2016, 463, 1518-1533. [CrossRef]

46. Sneden, C.; Roederer, I.U.; Boesgaard, A.M.; Lawler, J.E.; Den Hartog, E.; Cowan, J.J.; Sobeck, J. Detailed Iron-Group Abundances in a Very Metal-Poor Main Sequence Turnoff Star. Presented at the 2017 229th American Astronomical Society Meeting, Grapevine, TX, USA, 3-7 January 2017; p. 154.

47. Roederer, I.U.; Sneden, C.; Lawler, J.E.; Sobeck, J.S.; Cowan, J.J.; Boesgaard, A.M. Consistent Iron Abundances Derived from Neutral and Singly Ionized Iron Lines in Ultraviolet and Optical Spectra of Six Warm Metal-poor Stars. Astrophys. J. 2018, 860, 125:1-125:15. [CrossRef]

48. Wood, M.P.; Sneden, C.; Lawler, J.E.; Den Hartog, E.A.; Cowan, J.J.; Nave, G. Vanadium Transitions in the Spectrum of Arcturus. Astrophys. J. Suppl. Ser. 2018, 234, 25. [CrossRef]

49. Afşar, M.; Sneden, C.; Wood, M.P.; Lawler, J.E.; Bozkurt, Z.; Böcek Topcu, G.; Mace, G.N.; Kim, H.; Jaffe, D.T. Chemical Compositions of Evolved Stars From Near-Infrared IGRINS High-Resolution Spectra. I. Abundances in Three Red Horizontal Branch Stars. arXiv 2018, arXiv:astro-ph.SR/1808.03855.

50. Blackwell-Whitehead, R.J.; Lundberg, H.; Nave, G.; Pickering, J.C.; Jones, H.R.A.; Lyubchik, Y.; Pavlenko, Y.V.; Viti, S. Experimental TiI oscillator strengths and their application to cool star analysis. Mon. Not. R. Astron. Soc. 2006, 373, 1603-1609. [CrossRef]

51. Chavez, J.; Lambert, D.L. Isotopic Titanium Abundances in Local M Dwarfs. Astrophys. J. 2009, 699, $1906-1918$. [CrossRef]

(C) 2018 by the authors. Licensee MDPI, Basel, Switzerland. This article is an open access article distributed under the terms and conditions of the Creative Commons Attribution (CC BY) license (http://creativecommons.org/licenses/by/4.0/). 